\title{
Study of the relation between medial orbitofrontal artery and anterior skull base performed by computed tomography angiography*
}

\author{
Henrique C. Patricio', Alexandre Felippu², Carlos D. Pinheiro-Neto³, \\ Luiz U. Sennes ${ }^{4}$ \\ ' Otorhinolaryngology Department of the University of São Paulo (USP), SP and University of Extreme South of Santa Catarina \\ (UNESC), SC, Brazi \\ ${ }^{2}$ Felippu Institute of Otorhinolaryngology, SP, Brazi \\ Division of Otolaryngology/Head and Neck Surgery, Department of Surgery, Albany Medical Center, Albany, NY, USA \\ ${ }^{4}$ Otorhinolaryngology Department of the University of São Paulo (USP), SP, Brazil
}

Rhinology 56; 2: 172-177, 2018

https://doi.org/10.4193/Rhin17.121

*Received for publication:

June 8, 2017

Accepted: December 21, 2017

\begin{abstract}
Background: The aims of this study were to analyze the relationships between the medial orbitofrontal artery (MOFA) and the anterior skull base (ASB) including anatomical endonasal landmarks using computed tomography angiography (CTA).

Methods: We studied 52 CTAs using OsiriX ${ }^{\oplus}$ software. All CTAs were placed in the same anatomical position. MOFA was identified in the sagittal and coronal plane and its correlation with ASB was analyzed. The distance between the MOFA and landmarks for endonasal surgery were obtained, determining the high risk areas for its injury.
\end{abstract}

Results: After arising from the anterior cerebral artery, the MOFA dives inferiorly towards the ASB, close to the midline (average distance of $1.5 \mathrm{~mm}$ ), approaching the planum sphenoidale (average distance of $1.8 \mathrm{~mm}$ ) and then ascends away from the ASB as it runs anteriorly, with an average distance of $4.4 \mathrm{~mm}$ in the region of the anterior wall of the sphenoid sinus and $12 \mathrm{~mm}$ in the region of the anterior ethmoid artery.

Conclusions: The MOFA has an intimate relationship with the ASB and nasal cavity; the regions with the highest risk of surgical trauma are between the posterior ethmoid and the planum sphenoidale.

Key words: anatomy and histology, injuries, nasal surgical procedures, radiography, skull base

\section{Introduction}

With the advent of endoscopes in otolaryngology, systematization of functional endoscopic sinus surgery and popularization of the endoscopic techniques were seen worldwide ${ }^{(1-4)}$. The next step was the expansion of endoscopic techniques using the sinonasal cavity as a corridor to approach and resect skull base lesions. Consequently, surgical indications have considerably increased, as well as the severity of complications ${ }^{(5-10)}$. Among these complications, the two main causes of morbidity in endoscopic surgery of the skull base are vascular and/or cranial nerve injuries ${ }^{(11)}$. The medial orbitofrontal artery (MOFA) is the first cortical branch of the post-communicating segment of the anterior cerebral artery (ACA). It courses medially and inferiorly to the frontal lobe, supplying the straight gyrus, the medial and inferior part of the frontal lobe, the bulbs and olfactory tracts ${ }^{(12,13)}$. In case of dural violation during endoscopic sinus surgery, this artery can be injured leading to potential severe complications. Injuries of branches of the ACA after endoscopic endonasal surgery have been described in the literature, ranging from temporary to permanent sequelae, including death ${ }^{(11,14-16)}$. Computed tomography angiography (CTA) is a noninvasive exam that allows the diagnosis of vascular, skull base and neurological lesions ${ }^{(17)}$. It allows a good identification of the MOFA allowing the study of its relation with the base of the skull and nasal cavity. The CTA allows the study of the accurate trajectory of the artery, since there is no anatomical distortion that might happen with cadaveric dissection studies, for example. The aims of this study were to analyze the relationships between the MOFA and 


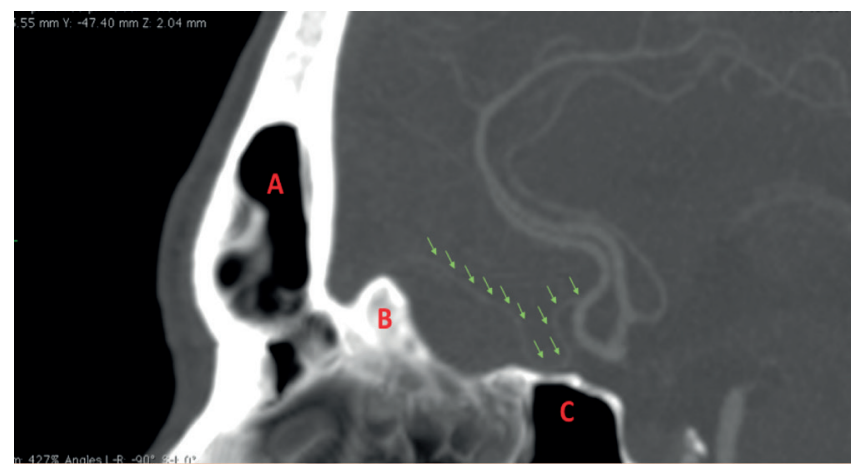

Figure 1. Computed tomography angiography in sagittal reconstruction, arrows marking the medial orbitofrontal artery. A (frontal sinus), B (ethmoidal crest) and C (sphenoidal sinus).

the anterior skull base (ASB) including anatomical endonasal landmarks using computed tomography angiograpy (CTA). Also to identify the sites of greater proximity of the MOFA to the ASB and establish risk areas for endonasal surgery.

\section{Materials and methods}

After approval by the ethic committee of the University of São Paulo School of Medicine, a prospective descriptive study of CTAs was performed at the Delboni Auriemo, Diagnostics of America SA Laboratory (DASA), from 2013 to 2015 in São Paulo - Brazil. Initially a total of 174 exams of adults of both sex were analyzed. Were discarded 122 exams by exclusion criteria: radiological signs of previous stroke, head trauma, craniofacial or cerebral surgery, craniofacial malformations, brain and sinonasal tumors, signs of hydrocephalus, intracranial hypertension, aneurysms, and exams where the MOFA was not adequately enhanced by the contrast. Fifty-two CTAs were included in the study. The MOFA was studied in the side where better contrast enhancement was obtained. The measurements were performed using Osiri ${ }^{\circledast}$ software in the chronological order in which the tests were acquired. Initially all the CTAs were placed in the same anatomical position, with the anterior skull base in the horizontal line and, in the sagittal plane, the MOFA was identified according to its anatomical description ${ }^{(12,13)}$ (Figure 1).

After delineation of the MOFA in the sagittal plane, the artery was found and marked in the coronal plane where the anterior ethmoidal foramen is identified. The software automatically locates the MOFA in the sagittal and axial planes. That was the first measurement point used and it was called point 1 (Figure 2).

In the coronal plane, measurements were taken between the MOFA and the midline, defined by an intracranial projection of the nasal septum position (measure $A$ ), between the MOFA and the nasal septum insertion in the ASB (measure B), between the MOFA and the insertion of the middle turbinate in the ASB (measure $C$ ) and between the MOFA to the closest point at the ASB with boundary to the sinonasal cavity (measure D) (Figure $3)$.

Measurements were also taken in the sagittal plane. The initial
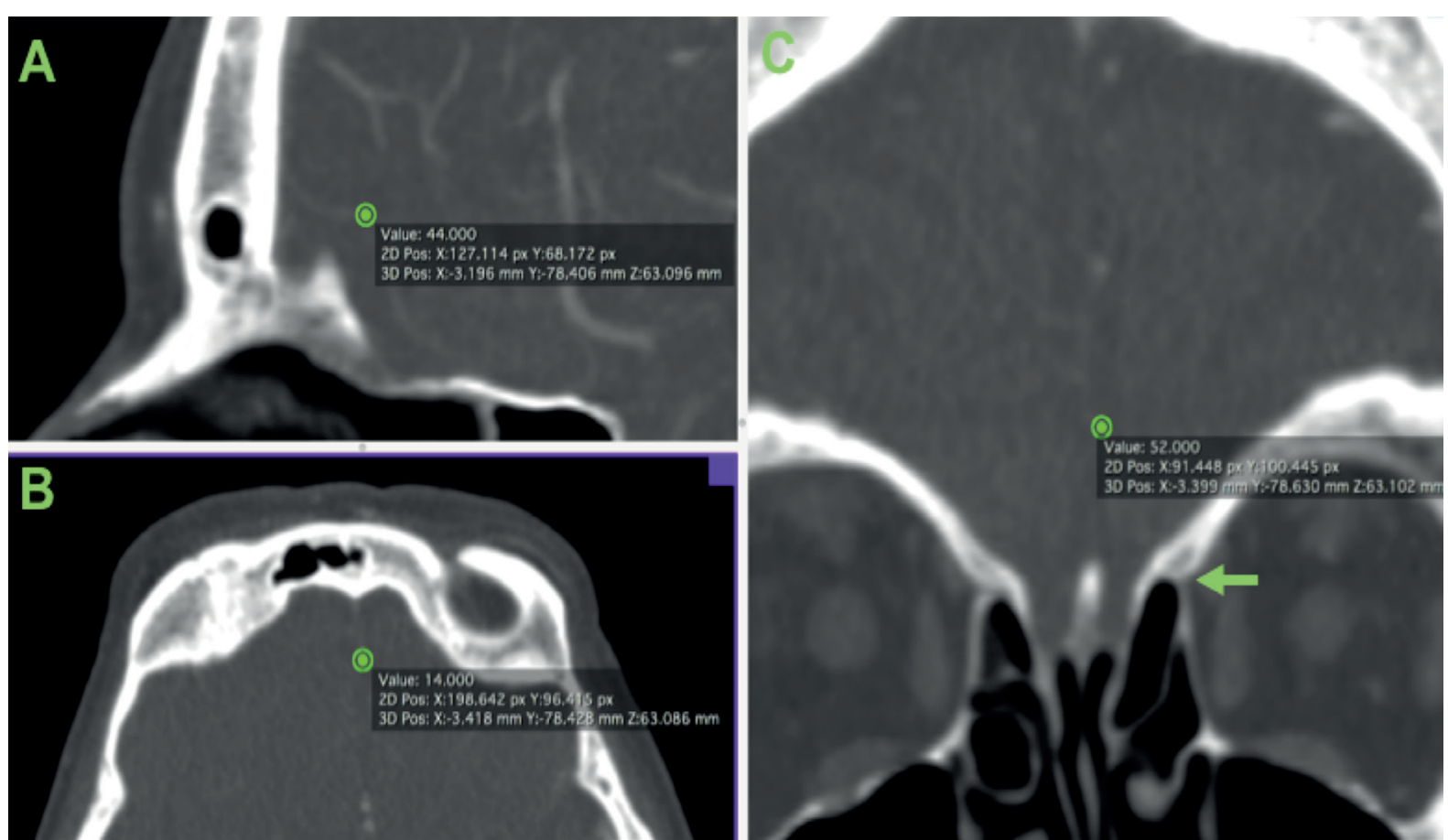

Figure 2. Computed tomography angiography showing point 1 (green circle) that identifies simultaneously the position of MOFA in the sagittal (A), axial (B) and coronal planes (C), at the level of anterior ethmoidal foramen (arrow). 


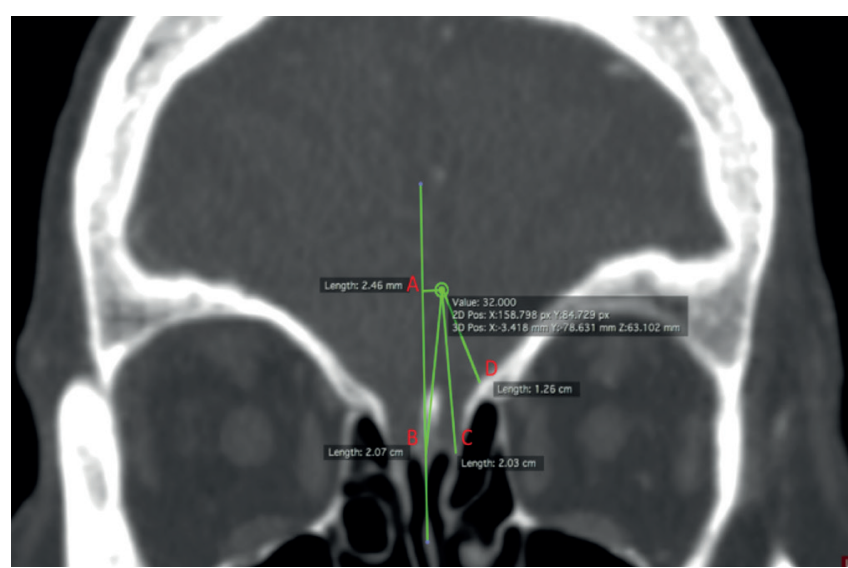

Figure 3. Computed tomography angiography in the coronal plane, exemplifying the measurements made between point 1 (green circle) and anatomical repairs: midline(A), insertion of the nasal septum in $A S B(B)$ insertion of the middle turbinate in $A S B(C)$ and the point of most proximity to the $A S B(D)$.

point of reference was the same point 1 and through it a linear and perpendicular line was drawn towards the ASB. The point where this perpendicular line meets the skull base/endonasal interface was called projection A (PA). The point where this perpendicular line encounters the crista galli was called projection $A^{*}(P A *)$. Point 2 was placed in the insertion of the anterior wall of the sphenoid sinus in the ASB, point 3 was placed in the tubercullum of the sella turcica and point 4 marks the origin of the MOFA in the pericallosal artery. Projection B (PB) was created at the midpoint between $P A$ and point 2. Projection $C(P C)$ was established between point 2 and point 3 . All of these points and projections had their distances measured to the point of most proximity to the MOFA, generating MOFA-A measurements (between MOFA and projection A), MOFA- $A^{*}$ (between MOFA and projection $A^{*}$ ), MOFA-2 (between MOFA and point 2 ), MOFA-PB (between MOFA and projection B), MOFA-3 (between MOFA and point 3), MOFA-PC (between MOFA and projection C). The distances between the MOFA point of origin (point 4) and points 2 and 3 were also measured, generating measurements 4-2 and 4-3, as shown in Figure 4.

In all exams the distance from the homolateral olfactory fossa to the MOFA was measured in the coronal plane where the anterior ethmoidal foramen is identified. The classification of Keros ${ }^{(18)}$ defines the olfactory fossa as type I (up to $3 \mathrm{~mm}$ ), type II (4 to 7 $\mathrm{mm}$ ) and type III (8 to $16 \mathrm{~mm}$ ) and was used for comparison.

For statistical analysis, the IBM software - SPSS (Statistical Package for the Social Sciences) version 20.0 was used. The values of the measurements of the variables were expressed in millimeters ( $\mathrm{mm}$ ): Average, standard deviation, minimum, maximum and confidence interval $(95 \% \mathrm{Cl})$. It was established

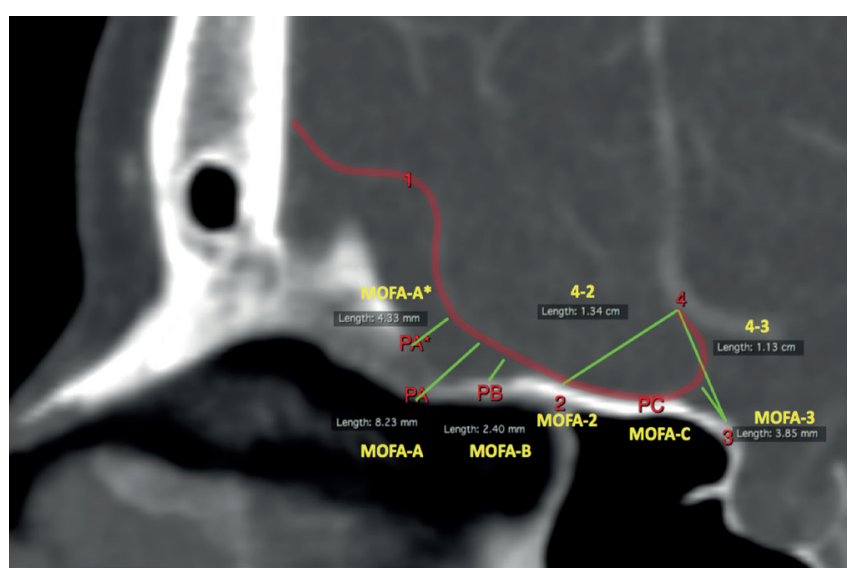

Figure 4. Computed tomography angiography in sagittal reconstruction exemplifying the shorter distance between MOFA (marked in red) and many references of anterior skull base: MOFA-A (shorter distance between MOFA and PA - projection A); MOFA-A* (between MOFA and $\mathrm{PA}^{*}$ - projection $\mathrm{A}^{*}$ ); MOFA-B (between MOFA and $\mathrm{PB}$ - projection $\mathrm{B}$ ); MOFA-C (between MOFA and PC - projection C); MOFA-2 (between MOFA and point 2); MOFA-3 (between MOFA and point 3). It also shows the distance between the origin of MOFA (point 4) and point 2: measure 4-2 and between point 4 and point 3: measure 4-3.

as significance level $p<0.05(a=0.05)$ and $95 \%$ confidence. For the comparison between the distance from the olfactory fossa to the MOFA and the Keros classification, the Wilcoxon T test was used. The correlation between the measurements and the values of Keros the Spearman correlation coefficient was selected.

\section{Results}

Among the 52 exams analyzed, $55.77 \%$ (29 patients) were male and $44.23 \%$ ( 23 patients) female. The average age was 52 years old (range between 24 and 86 years). As for laterality, 71.15\% (37 exams) were measured on the left side and $28.85 \%$ (15 patients) on the right side. Only 17 exams were excluded because the MOFA was not adequately enhanced by the contrast. Table 1 summarizes the coronal plane measurements and Table 2 shows the sagittal plane measurements.

The distance between MOFA and ASB in the coronal plane (measurements $A, B, C$ and $D$ ) had no statistical significance compared to the Keros classification.

Using the averages between the reference points and the MOFA, it was possible to draw a sketch of the trajectory of the artery at the points studied, as shown in Figures 5.

\section{Discussion}

With the technological advancement, the imaging tests have also become a tool for the anatomical studies. CTA is a noninvasive exam and widely available in the clinical practice 
Table 1. Distance $(\mathrm{mm})$ between point 1 and the different anatomical repairs of anterior skull base in the coronal plane $(n=52)$.

\begin{tabular}{cccccccc|}
\hline $\begin{array}{c}\text { Mea- } \\
\text { sures }\end{array}$ & $\begin{array}{r}\text { Ave- } \\
\text { rage }\end{array}$ & SD & $\begin{array}{c}\text { Mini- } \\
\text { mum }\end{array}$ & $\begin{array}{c}\text { Maxi- } \\
\text { mum }\end{array}$ & $\begin{array}{r}\text { Me- } \\
\text { dium }\end{array}$ & CV (\%) \\
\hline A & 1,5 & 1,2 & 0,0 & 5,6 & 1,0 & 85,73 \\
\hline B & 15,9 & 6,7 & 5,3 & 36,5 & 16,4 & 42,49 \\
\hline C & 15,9 & 6,5 & 5,1 & 35,9 & 16,7 & 40,88 \\
\hline D & 11,5 & 5,2 & 2,0 & 26,8 & 11,1 & 45,14 \\
\hline
\end{tabular}

Measure A: distance from point 1 to a projection of the sagittal plane in the median line. Measure $B$ : distance from point 1 to the insertion of the nasal septum at the base of the skull. Measure C: distance from point 1 to the insertion of the middle turbinate at the base of the skull. Measure D: distance from point 1 to the point of greatest proximity to the base of the skull. SD: Standard Deviation; CV = coefficient of variability.

for pre-operative planning. In some services, CTA is routinely obtained prior to endoscopic endonasal transcribriform and/or transplanum approaches. This exam allows 3D visualization and evaluates not only the vessel but also its relation with bone and brain structures ${ }^{(19,20)}$. In the 174 standard cranial CTA exams initially analysed, MOFA was identifyed in more then $90 \%$ of cases. An especific protocol for MOFA identification might increase this sensibility, once it is consistently found bilaterally in anatomical studies ${ }^{(21-23)}$. The study of artery position in cranial CTA avoid possible anatomical distortion that might happen during cadaveric dissection, since the mobilization of encephalic structures or removal of endonasal structures could modify the position of the artery. The studies published in the literature regarding the MOFA used cadaver dissection and did not correlate the MOFA to skull base landmarks ${ }^{(13,21,24)}$. These studies described MOFA as the smallest of the cortical branches of the ACA, ranging in diameter from 0.21-1.85 millimeters (average of 0.79 millimeters) ${ }^{(13)}$, originating from the segment $A 2$ of the ACA, proceeding forward and downward in the direction of the tract and olfactory bulb. The MOFA was medial to the straight gyrus, necessitating dissection of the interhemispheric fissure to identify its origin, close to the anterior communicating complex ${ }^{(21)}$. We verified that the average distance from the MOFA to the midline was 1.5 $\mathrm{mm}$ at point 1 (coronal plane at the level of anterior ethmoidal) showing that it remains close to the interhemispheric fissure. This position near the midline demonstrates an area of greater risk of trauma in endonasal surgery, due to the fragility of the ethmoidal bone in the midline, especially in the cribriform plate and olfactory fossa. We have also confirmed the trajectory downward and forward of the MOFA until it reaches the planum sphenoidale, which is the point of greatest proximity of the ASB, with an average distance of $1.8 \mathrm{~mm}$. In 26 cases (50\%), the MOFA
Table 2. Shorter distance $(\mathrm{mm})$ between the MOFA and the different anatomical repairs of anterior skull base in the sagittal plane $(n=52)$.

\begin{tabular}{|ccccccc|}
\hline Local & $\begin{array}{r}\text { Ave- } \\
\text { rage }\end{array}$ & SD & $\begin{array}{c}\text { Mini- } \\
\text { mum }\end{array}$ & $\begin{array}{r}\text { Maxi- } \\
\text { mum }\end{array}$ & $\begin{array}{r}\text { Me- } \\
\text { dium }\end{array}$ & CV \\
\hline MOFA-A & 12,0 & 5,8 & 2,6 & 33,9 & 13,1 & 48,19 \\
\hline MOFA-A* & 10,2 & 6,4 & 0,0 & 33,9 & 10,3 & 62,74 \\
\hline MOFA-2 & 4,4 & 3,2 & 0,0 & 10,7 & 4,6 & 73,32 \\
\hline MOFA-3 & 5,6 & 3,0 & 0,0 & 14,2 & 5,2 & 53,85 \\
\hline MOFA-B & 6,9 & 4,2 & 0,0 & 15,2 & 8,0 & 61,73 \\
\hline MOFA-C & 1,8 & 2,9 & 0,0 & 15,2 & 0,3 & 161,1 \\
\hline $4-2$ & 16,0 & 4,4 & 8,2 & 26,4 & 16,4 & 27,57 \\
\hline $4-3$ & 10,2 & 3,0 & 3,8 & 16,2 & 9,9 & 29,81 \\
\hline
\end{tabular}

MOFA-A: shorter distance between MOFA and skull base in projection A. MOFA-A*: shorter distance between MOFA and base of skull in projection $A^{*}$. MOFA-2: shorter distance between the MOFA and the skull base at point 2. MOFA-3: shorter distance between the MOFA and the skull base at point 3. MOFA-B: less distance between the MOFA and the skull base in projection B. MOFA-C: less distance between MOFA and base of skull in projection C. 4-2: shorter distance between point 4 and base of skull in point 2. 4-3: shorter distance between point 4 and the skull base at point 3. SD: Standard Deviation; CV = coefficient of variability.

was so close to the planum sphenoidale that its distance could not be measured. From the planum sphenoidale the MOFA followed an ascending curve away from the ASB. Average distance of $4.4 \mathrm{~mm}$ from the junction of the anterior wall of the sphenoid sinus and the ASB; and $12 \mathrm{~mm}$ distant from the ASB at the level of the anterior ethmoidal foramen (the most anterior site measured in the study). At this point, 22 of the 52 exams analyzed presented interposition of the crista galli between the MOFA and the ASB, with MOFA being on average $6 \mathrm{~mm}$ closer to the crista galli than to the ASB. The potential risk of MOFA injury is a reality that many surgeons have overlooked, with limited literature on the subject. But some authors mentioned severe complications following endonasal endoscopic surgery associated with ASB lesion and intracranial hemorrhage. Maniglia (1991) ${ }^{(14)}$ reported a case that evolved to death after traumatic injury to ASB and intracranial bleeding, due to injury to the anterior cerebral artery. Berenholz (1999) ${ }^{(15)}$ reported a case of vascular injury during ethmoidectomy with microdebrider for the treatment of nasal polyposis. The patient had a subarachnoid hemorrhage and small infarction of the frontal lobe, probably due to lesion of branches of the ACA. Tawadros and Prahlow (2008) ${ }^{(16)}$ described a case that evolved to death after an endoscopic sinus surgery for chronic rhinosinusitis associated with nasal polyposis. There was a lesion of the cribriform plate, dura mater, olfactory nerve 


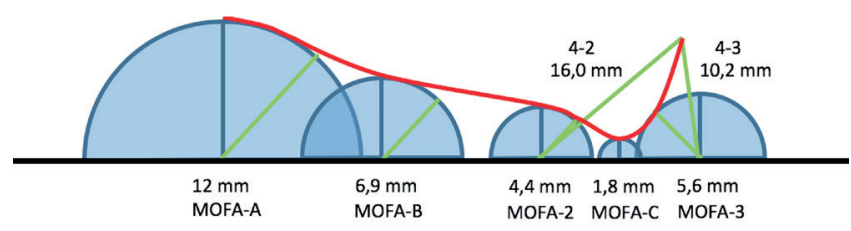

Figure 5. Graphical representation of the possible average position of the MOFA (red line), according to its proximity to the skull base, hypothetically referred to as rectilinear (black line), at the sites measured in the study: measurements (MOFA-A, MOFA-B, MOFA-2, MOFA-C, MOFA-3, 4-2 and 4-3) respectively represented by the green line.

and subarachnoid hemorrhage resulting from injury to the ACA. Al-Afif (2017) ${ }^{(25)}$ reported four patients with iatrogenic perforating cerebral lesions after routine functional endoscopic sinus surgery. In three of them cerebral infarction developed secondary to injury of branches of the anterior cerebral artery. Two had rapid global brain swelling and they succumbed within days and the other two patients survived without apparent neurological deficits. We have demonstrated that the proximity of the MOFA to the cranial base and its position near to the midline makes it the first large intracranial vessel that can be potentially injured during an endonasal surgical procedure. This relationship is even closer in the posterior ethmoid and planum sphenoidale, making them as areas at greater risk of MOFA injury. Felippu (2011) (26) suggests that after identification of ASB during ethmoidectomy the endoscope of 30 degrees should be turned down before following the dissection towards the sphenoid sinus. In case the surgeon does not correct his position during the ethmoidectomy or disorientation occurs, there is increased risk of ASB injury. During endoscopic ASB surgery rate of complications reaches $17.1 \%$ and the iatrogenic CSF fistula is one of the most common complications ${ }^{(11,26)}$. The area of greater risk of iatrogenic cerebrospinal fluid leak is the anterior ethmoid region (28) and the probability of MOFA lesion in this site is lower, considering the mean distance of $11.5 \mathrm{~mm}$ from the MOFA to ASB at the level of the anterior ethmoidal artery.

We have created 4 risk zones, based on the proximity of the MOFA to the ASB and the working angle in the transnasal endoscopic surgery (Figure 6). Zone 1 (low risk) corresponds to the area anterior to the anterior ethmoidal artery. Zone 2 (medium risk) extends from the anterior ethmoidal artery to the beginning of the posterior ethmoid. Zone 3 (high risk) involves final part of the posterior ethmoid and includes the planum sphenoidale. Zone 4 (medium risk) corresponds to the tuberculum sellar. The technological evolution of the endoscopes, imaging equipments, and neuronavigation provided a better understanding of the anatomy of the sinuses, making a surgical corridor for more hidden and deeper regions of the skull base ${ }^{(9,29-31)}$. The limits of these skull base accesses have not yet been established and although much is known about the anatomy of ASB,

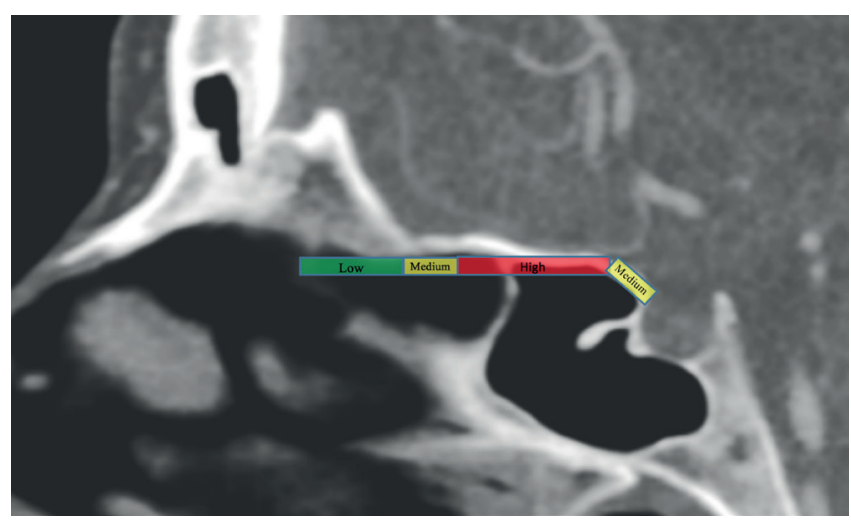

Figure 6. Computed tomography angiography image in sagittal reconstruction showing the 4 risk zones exemplified by green (low), yellow (medium) and red (high), according to the proximity of the MOFA to the anterior skull base.

little knowledge exists about its relationships with endonasal neurovascular structures ${ }^{\left({ }^{32}\right)}$. Our study contributes to increase this knowledge, verifying the position of the MOFA in relation to ASB. According to the 4 risk zones, we suggest that the risk of MOFA injury is lower in transfrontal and transsellar approaches than in transcribriform and transplanum approaches. Kassam (2011) ${ }^{(11)}$ analyzing the skull base endonasal endoscopic surgeries of the 800 patients confirmed this concern, especially in the transcribriform approach, where it reports that among the most important structures of this access is the segment A2 of the anterior cerebral artery and its frontal and medial orbitofrontal branches. Even tough injury to the MOFA during functional endoscopic sinus surgery is rare nowadays, this artery should be considered during every anterior cranial base resection. In cases of sinonasal pathology with minimal intracranial extension, the anatomy of the MOFA should be mostly intact. This is commonly seen in cases of esthesioneuroblastomas and other sinonasal malignancies where the anterior cranial base dura and/or olfactory bulbs are not grossly involved but are still resected for margins. In the other hand, intracranial pathologies like meningiomas very often alter the anatomy of the MOFA and the CTA is extremely important to define the position of the artery in relation to the tumor. Not uncommonly, the MOFA is encased in the tumor which makes the resection more challenging.

\section{Conclusion}

The MOFA dives inferiorly towards the ASB, close to the midline (average distance of $1.5 \mathrm{~mm}$ ), approaching the planum sphenoidale (average distance of $1.8 \mathrm{~mm}$ ) and then ascends away from the ASB as it runs anteriorly, with an average distance of $4.4 \mathrm{~mm}$ in the region of the anterior wall of the sphenoid sinus and 12 $\mathrm{mm}$ in the region where the anterior ethmoid artery is located. There was no relation between the MOFA position and the Keros Classification. Considering the angle of work in endonasal 
surgery and the places of greater proximity of the MOFA to the anterior skull base, the regions of the posterior ethmoid and planum sphenoidale were the areas of greatest risk.

\section{Acknowledgement}

Statistical analysis: Rocha F. R. (Department of biostatistics, University of the Extreme South of Santa Catarina - UNESC, Criciúma - Santa Catarina, Brazil).

\section{Authorship contribution}

HCP: study design, data collection and analysis, manuscript production; AF: study design, manuscript review; CDPN: manuscript review; LUS: study design, manuscript review

\section{Conflict of interest}

The authors declare no conflicts of interest.

\section{References}

1. Messerklinger W. Background and evolution of endoscopic sinus surgery. Ear Nose Throat J. 1994 Jul;73(7):449-50.

2. Wigand ME. Transnasal ethmoidectomy under endoscopical control. Rhinology. 1981 Mar;19(1):7-15.

3. Stammberger $\mathrm{H}$, Posawetz W. Functional endoscopic sinus surgery. Concept, indications and results of the Messerklinger technique. Eur Arch Otorhinolaryngol. 1990;247(2):63-76.

4. Kennedy DW. Technical innovations and the evolution of endoscopic sinus surgery. Ann Otol Rhinol Laryngol Suppl. 2006 Sep;196:312.

5. Jho HD, Carrau RL. Endoscopic endonasal trans sphenoidal surgery: experience with 50 patients. J Neurosurgery. 1997 Jul;87(1):44-51.

6. Stammberger $\mathrm{H}$, Anderhuber W, Walch $\mathrm{C}$ Papaefthymiou G. Possibilities and limitations of endoscopic management of nasal and paranasal sinus malignancies. Acta Otorhinolaryngol Belg. 1999;53(3):199-205.

7. Thaler ER, Kotapka M, Lanza DC, Kennedy DW. Endoscopically assisted anterior cranial skull base resection of sinonasal tumors. Am J Rhinol. 1999 Jul-Aug; 13(4):303-10.

8. Kassam A, Snyderman CH, Mintz A, Gardner P, Carrau RL. Expanded endonasal approach: the rostrocaudal axis. Part I. Crista galli to the sella turcica. Neurosurg Focus. 2005 Jul 15;19(1):E3.

9. Cavallo LM, Messina A, Cappabianca P, Esposito F, de Divitiis E, Gardner P, Tschabitscher M. Endoscopic endonasal surgery of the midline skull base: anatomical study and clinical considerations. Neurosurg Focus. 2005 Jul 15;19(1):E2.

10. Snyderman $\mathrm{CH}$, Kassam AB. Endoscopic techniques for pathology of the anterior cranial fossa and ventral skull base. J Am Coll Surg. 2006 Mar;202(3):563.

11. Kassam AB, Prevedello DM, Carrau RL, Snyderman CH, Thomas A, Gardner $P$, Zanation A, Duz B, Stefko ST, Byers K, Horowitz MB. Endoscopic endonasal skull base surgery: analysis of complications in the authors' initial 800 patients. J Neurosurg. 2011 Jun;114(6):1544-68.

12. Rhoton $\mathrm{AL} \mathrm{Jr}$. The anterior and middle cranial base. Neurosurgery. 2002 Oct;51(4 Suppl):S273-302.

13. Avci E, Fossett D, Aslan M, Attar A, Egemen $\mathrm{N}$. Branches of the anterior cerebral artery near the anterior communicating artery complex: an anatomic study and surgical perspective. Neurol Med Chir (Tokyo). 2003 Jul;43(7):329-33.

14. Maniglia AJ. Fatal and other major complications of endoscopic sinus surgery. Laryngoscope. 1991 Apr;101(4 Pt 1):349-54.

15. Berenholz L, Kessler A, Sarfaty S, Segal S Subarachnoid hemorrhage: a complication of endoscopic sinus surgery using powered instrumentation. Otolaryngol Head Neck Surg. 1999 Nov;121(5):665-7.

16. Tawadros AM, Prahlow JA. Death related to nasal surgery: case report with review of therapy-related deaths. Am J Forensic Med Pathol. 2008 Sep;29(3):260-4.

17. Harbaugh RE, Schlusselberg DS, Jeffery R, Hayden S, Cromwell LD, Pluta D, English RA. Three-dimensional computed tomographic angiography in the preoperative evaluation of cerebrovascular lesions. Neurosurgery. 1995 Feb;36(2):320-6.

18. Keros P. [On the practical value of differences in the level of the lamina cribrosa of the ethmoid]. Z Laryngol Rhinol Otol. 1962 Nov;41:809-13.

19. Schwartz RB, Tice HM, Hooten SM, Hsu L, Stieg PE. Evaluation of cerebral aneurysms with helical CT: correlation with conventional angiography and MR angiography Radiology. 1994 Sep;192(3):717-22.

20. Katz DA, Marks MP, Napel SA, Bracci PM, Roberts SL. Circle of Willis: evaluation with spiral CT angiography, MR angiography, and conventional angiography. Radiology. 1995 May;195(2):445-9

21. Mavridis IN, Kalamatianos T, Koutsarnakis C, Stranjalis G. The Microsurgical Anatomy of the Orbitofrontal Arteries. World Neurosurg. 2016 May;89:309-19.

22. Araujo Filho BC, Pinheiro-Neto CD, Ramos HF, Voegels RL, Sennes LU. [Endoscopic ligation of the anterior ethmoidal artery: a cadaver dissection study]. Braz J Otorhinolaryngol. 2011 Jan-Feb;77(1):33-8.

23. Lee WC, Ming Ku PK, van Hasselt CA. New guidelines for endoscopic localization of the anterior ethmoidal artery: a cadaveric study. Laryngoscope. 2000 Jul;110(7):11738.

24. Kedia S, Daisy S, Mukherjee KK, Salunke $P$, Srinivasa R, Narain MS. Microsurgical anatomy of the anterior cerebral artery in Indian cadavers. Neurol India. 2013 MarApr;61(2):117-21.

25. Al-Afif S, Hermann EJ, Majernik GH,
Nakamura M, Raab P, Lenarz T, Krauss JK. Severe cerebral complications secondary to perforation injury of the anterior skull base during sinonasal surgery: an underappreciated problem? World Neurosurg. 2017 Sep 21.

26. Felippu A. Nasal centripetal endoscopic sinus surgery. Ann Otol Rhinol Laryngol. 2011 Sep;120(9):581-5.

27. Borg A, Kirkman MA, Choi D. Endoscopic Endonasal Anterior Skull Base Surgery: A Systematic Review of Complications During the Past 65 Years. World Neurosurg. 2016 Nov; $95: 383-391$

28. Araujo Filho BC, Butugan O, Pádua FG, Voegels RL. Endoscopic repair of CSF rhinorrhea: experience of 44 cases. Braz $J$ Otorhinolaryngol. 2005 Jul-Aug;71(4):472-6.

29. Langdon C, et al. Expanded endoscopic endonasal surgery for advanced stage juvenile angiofibromas: a retrospective multi-center study. Rhinology. 2016 Sep;54(3):239-46.

30. Castelnuovo P, Dallan I, Battaglia P, Bignami M. Endoscopic endonasal skull base surgery: past, present and future. Eur Arch Otorhinolaryngol. 2010 May;267(5):649-63.

31. Bastier PL, de Gabory L. Design and assessment of an anatomical diagram for sinonasal malignant tumour resection. Rhinology. 2016 Dec 1;54(4):361-367.

32. Snyderman $\mathrm{CH}$, Pant $\mathrm{H}$, Carrau $\mathrm{RL}$, Prevedello D, Gardner P, Kassam AB. What are the limits of endoscopic sinus surgery? the expanded endonasal approach to the skull base. Keio J Med. 2009 Sep;58(3):15260.

Henrique C. Patricio

Otorhinolaryngology - University of São Paulo Medical School (USP).

Graduation course in medicine University of the Extreme South of Santa Catarina Medical School Santa Catarina

Brazil

E-mail:

henriquepatricio@hotmail.com 